\title{
Delayed Bleeding as a Complication of Kidney Biopsy
}

\author{
Heiko Hickstein ${ }^{\mathrm{a}}$, Jan Stange ${ }^{\mathrm{b}}$, Sebastian Koball ${ }^{\mathrm{b}, \mathrm{c}}$
}

\begin{abstract}
We report the case of a 60 years old female treated because of fluid lung under the presumption of acute oliguric renal failure in decompensated diabetic nephropathy with dialysis via a central venous line. Normal creatinine test results before admission were the reason for further diagnostics. Positive P-ANCA and Anti-MPOAntibodies and the result of the renal biopsy proved the diagnosis of a renal limited microscopic polyangiitis. An immunosuppressive therapy with methylprednisolone and cyclophosphamide was initiated and a course of plasma exchange with substitution of ringeralbumin 5\%- solution was started. Four days after biopsy a clinical deterioration occurred. The sonography image showed no clear result - an after that performed CT scan confirmed the diagnosis of an acute retroperitoneal bleeding. We recommend in these cases a partial or complete use of fresh frozen plasma as substitution fluid for plasma exchange and under suspicion of retroperitoneal bleeding a CT scan as the imaging modality of choice.
\end{abstract}

Keywords: Kidney biopsy; Bleeding; Complications

\section{Case}

A 60 years female was admitted to our hospital because of cardiac decompensation (NYHA III) and corresponding renal failure with oliguria.

Because of progressive hyperkalemia (serum-potassium $6.3 \mathrm{mmol} / \mathrm{L}$ ) and uraemia (creatinine $766 \mu \mathrm{mol} / \mathrm{L}$ (35 - 88

Manuscript accepted for publication June 12, 2012

${ }^{a}$ Kuratorium fur Heimdialyse, Wismar, Germany

${ }^{b}$ Div. of Nephrology, University of Rostock Department of Internal Medicine, Germany

${ }^{\mathrm{c} C o r r e s p o n d i n g ~ a u t h o r: ~ S e b a s t i a n ~ K o b a l l, ~ U n i v e r s i t y ~ o f ~ R o s t o c k, ~}$ Department of Internal Medicine-Nephrology, Ernst-Heydemann-Street 6, 18055 Rostock, Germany. Email: Sebastian.koball@uni-rostock.de

doi:10.4021/wjnu16w $\mu \mathrm{mol} / \mathrm{L})$, urea $55 \mathrm{mmol} / \mathrm{L}(2.5$ - $6.1 \mathrm{mmol} / \mathrm{L})$, emergency dialysis therapy was initiated via a central venous catheter.

The patient had an underlying combined post-rheumatic aortic-mitral-valve dysfunction which had been treated with valve replacement with anticoagulation therapy since 12 years and pulmonary hypertension with insufficiency of the tricuspidal valve grade IV (PAPS $80 \mathrm{mmHg}$ ) and insulin dependent diabetes mellitus type II.

As the patient also reported joint pain in changing locations and was known to have stable renal function 4 weeks before the admission (serum creatinine at this time had been $139 \mu \mathrm{mol} / \mathrm{L}$ ), the diagnostic program was extended, although the clinical condition improved with dialysis, which was initially started under the presumption of end stage renal disease in a diabetic patient.

Renal biopsy performed 7 days after admission revealed a diffuse extracapillary focal necrotizing pauci immune glomerulonephritis with atrophic tubuli and interstitial fibrosis.

The immunological markers ANA 1:1.280 (norm value 1: < 320), p-ANCA 1: > 20.480 (norm value $<1: 10$ ) and Anti-MPO-Antibodies $(2390 \mathrm{U} / \mathrm{mL})$ (normal $<6.0 \mathrm{U} / \mathrm{mL}$ ) pointed at the diagnosis of a rapid-progressive GN with microscopic polyangiitis.

The patient received one dose $500 \mathrm{mg}$ Endoxan iv and Methylprednisolon $500 \mathrm{mg}$ iv per day for a total of 3 days, followed by a decreasing dose regimen starting at $1.0 \mathrm{mg} / \mathrm{kg}$.

At day 9, a therapeutic plasma exchange of $3.000 \mathrm{~mL}$ was performed, using 5\% albumin in ringer-lactate. A second plasma exchange was done on day 10 and on day 11 a haemodialysis treatment. After this therapy set, the ANA were reduced to $1: 160$ and the Anti-MPO-Abs to $575 \mathrm{U} / \mathrm{mL}$.

At the evening of day 11 the patient complained about pain in her left side at the entry of the biopsy needle. Ultrasound diagnostic revealed an inhomogeneous structure of the punctured kidney, reminiscent of inflammatory changes. Free fluid around the kidney as an indicator for bleeding was not seen. Therefore, the third plasma exchange was performed on day 12.

Due to hemodynamic instability coupled with decrease of the haemoglobin value, the third plasma exchange was interrupted prematurely and CT scan was initiated, which revealed an extended inhomogeneous mass around the left 


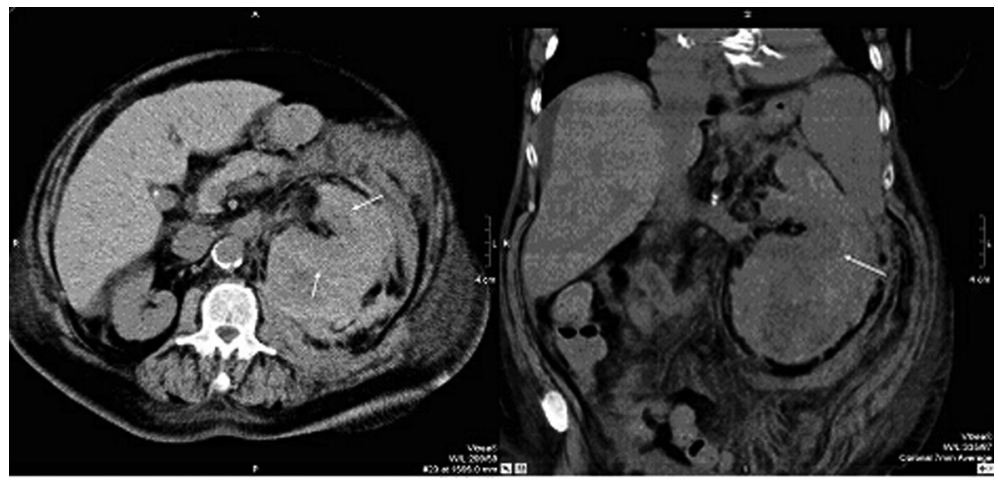

Figure 1. Computer tomography of abdomen performed 5 days after renal biopsy: the transversal and coronary sectional image shows the haematoma, the arrows demarcate the kidney.

kidney which pointed at a combination of parenchymal and subcapsular bleedings. There was also free fluid beneath the spleen and in the lower abdomen (Fig. 1).

Because of the co morbidities and the relatively high operational risks the patient underwent intensive care with transfusion (16 packed red blood cells and 6 packs of fresh frozen plasma) rather than surgery. Continuous heparin for anticoagulation was applied for protection of the mechanic heart valves. After stabilization of the patient, the patient received an arteriovenous fistula and was discharged into a tertiary care centre under continued intermittend dialysis.

The patient passed away 4 weeks later (day 51) due to cardiac insufficiency.

\section{Discussion}

Plasma exchange (PE) has been advocated in the therapy of many autoimmune diseases, especially in severe cases and might have a role in ANCA-associated glomerulonephritis. However, most of the studies reported benefit only in a few conditions, but also included a heterogeneous mix of patients with a variety in natural history and response to immunosuppressive therapy [1].

In the MEPEX-study (methylprednisolone versus plasma exchange for severe renal vasculitis) it was shown, that renal failure at presentation carries an increased risk for ESRD and death despite immunosuppressive therapy and the addition of plasma exchange (PE) was more effective than intravenous methylprednisolone (MEP) in the achievement of renal recovery in those who presented with a serum creatinine $>500 \mu \mathrm{mol} / \mathrm{L}(5.8 \mathrm{mg} / \mathrm{dL})$. In a total of 137 patients with ANCA-associated systemic vasculitis at 3 months $49 \%$ after intravenous MEP compared to $69 \%$ after PE who were alive and independent of dialysis. Plasma exchange was associated with a reduction of risk for progression to ESRD, from $43 \%$ in the MEP-group to $19 \%$ in the PE-group at 12 months. Hence, plasma exchange increased the rate of renal recovery in ANCA-associated systemic vasculitis which presented initially with renal failure when compared with intravenous methylprednisolone. Patient survival and severe adverse event rates were similar in both groups [2].

In the present case, the documentation of compensated renal function prior to the admission in combination with the elevated antibody titres led to the decision to apply plasma exchange with prednisolone therapy in the hope to recover renal function.

The initial plasma exchange therapies were effective in reducing the antibodies, however, severe bleeding complications forced interruption of the therapy, and therefore a beneficial clinical effect is speculative and unproven.

However, the present case shows, that even in elderly diabetics the possibility of an acute event, as vasculitis, as the cause of renal failure can not be ruled out and also in those patients diagnostic for acute autoimmune vasculitis should be considered. It is always important, to research prior creatinine course from the referring physicians.

Another lesson to learn is, that even 4 days after a renal biopsy, bleeding can still occur as a complication. Unconfirming ultrasound diagnostic does not safely exclude retroperitoneal or subcapsular bleedings. This imaging modality has in dependence on investigator and investigative conditions low sensitivity in detection of renal injuries and overlooks significant damages [3]. Thus in patients who have clear indications for renal imaging, CT scan is the modality of choice.

In patients with enhanced bleeding risks (e.g. due to anticoagulation for heart valve protection), an additional deterioration of the coagulation system due to plasma exchange against human albumin solutions should also be considered. Preferably, in those patients plasma may be exchanged against mixtures of fresh frozen plasma and albumin

\section{Acknowledgement}

The authors declare the absence of any financial support or relationship that may pose a conflict of interest. 


\section{References}

1. Gaskin G, Pusey CD. Plasmapheresis in antineutrophil cytoplasmic antibody-associated systemic vasculitis. Ther Apher. 2001;5(3):176-181.

2. Jayne DR, Gaskin G, Rasmussen N, Abramowicz D, Ferrario F, Guillevin L, Mirapeix E, et al. Randomized trial of plasma exchange or high-dosage methylprednisolone as adjunctive therapy for severe renal vasculitis. $\mathrm{J}$ Am Soc Nephrol. 2007;18(7):2180-2188.

3. Jalli R, Kamalzadeh N, Lotfi M, Farahangiz S, Salehipour $\mathrm{M}$. Accuracy of sonography in detection of renal injuries caused by blunt abdominal trauma: a prospective study. Ulus Travma Acil Cerrahi Derg. 2009;15(1):23-27. 